\title{
Mental disorders, disability and treatment gap in a protracted refugee setting ${ }^{\dagger}$
}

Augusto E. Llosa, Zeina Ghantous, Renato Souza, Fabio Forgione, Pierre Bastin, Alison Jones, Annick Antierens, Andrei Slavuckij and Rebecca F. Grais

\section{Background}

Studies have shown high levels of distress and mental disorder among people living in refugee camps, yet none has confirmed diagnosis through clinical reappraisal.

\section{Aims \\ To estimate the prevalence of mental disorders, related disability and treatment gap in adult refugees living in the Burj el-Barajneh camp.}

\section{Method}

Randomly selected participants were screened by household representative $(n=748)$ and individual $(n=315)$ interviews; clinical reappraisal was performed on a subset $(n=194)$ of 326 selected participants. Weighted prevalence estimates and $95 \%$ confidence intervals were calculated.

\section{Results}

The prevalence of current mental disorders was $19.4 \%$
(95\% Cl 12.6-26.2); depression was the most common diagnosis $(8.3 \%, 95 \% \mathrm{Cl} 4.4-12.2)$ and multiple diagnoses were common (42\%) among the 88 persons with mental disorder. Lifetime prevalence of psychosis was 3.3\% (95\% Cl 1.0-5.5). Mental disorders were associated with moderate to severe dysfunction (odds ratio $=8.8,95 \%$ Cl 4.5-17.4). The treatment gap was 96\% (95\% Cl 92-100).

\section{Conclusions}

A range of mental disorders and associated disability are common in this long-term refugee setting. Combined with an important treatment gap, findings support the current consensus-based policy to prioritise availability of mental health treatment in refugee camps, especially for the most severe and disabling conditions.

\section{Declaration of interest}

None.
More than 40 million people are currently displaced by war and persecution, with over 7 million living in temporary camps for over a decade. ${ }^{1}$ People who have survived violent conflict are at increased risk of mental health problems. Deleterious socioeconomic and material conditions often associated with forced displacement, including relative poverty, absence of healthcare and destruction of social networks, modulate this susceptibility and place those most vulnerable at risk of neglect and abuse. ${ }^{2-5}$ Furthermore, the treatment gap for even the most severe mental disorders is large and is most pronounced in lower-income settings. ${ }^{6}$

The Burj el-Barajneh camp was established in a southern suburb of Beirut in 1948 and hosts close to 20000 refugees in crowded, precarious conditions. Social and economic exclusion contribute to the high levels of poverty. ${ }^{7,8}$ Mental healthcare spending in Lebanon is mainly out-of-pocket and consequently least affordable to those with lower incomes, such as refugees. ${ }^{9}$ Prior studies have shown a high prevalence of distress and depression in vulnerable groups in Lebanon. These studies, however, have relied on unconfirmed, self or proxy reports and focused on distress and depression, mostly in women. Moreover, there is a growing interest in understanding the breadth of mental health outcomes, beyond the widely reported post-traumatic stress disorder (PTSD), in post-conflict and other humanitarian settings; in particular, little is known about psychotic disorders in these contexts. ${ }^{2,4,10}$ Here we estimate, with confirmatory clinical assessment, the prevalence of severe and common mental disorders, disability and treatment gap in adult refugees from Palestine living in the Burj el-Barajneh camp.

'See editorial, pp. 176-177, this issue.

\section{Method}

The study proposal was approved by camp authorities, community leaders, the Médecins Sans Frontières (MSF) ethics review board and the Lebanese American University Committee on Human Subjects in Research. Inclusion in the survey was voluntary and required written informed consent. Médecins Sans Frontières Operational Centre Geneva has provided free mental healthcare to the population of Burj el-Barajneh since early 2009, and was handed over to local partners in December 2012. All participants and non-participants were offered information and encouraged to visit MSF services that were provided within and outside the camp. Where requested, social service home visits and referrals to MSF or other organisations were provided.

\section{Participants}

This cross-sectional study used two-phase or double-sampling method for diagnostic prevalence determination. This method allows greater accuracy and efficiency in detecting less common conditions. ${ }^{11,12}$ Interviews took place between July and November 2010. Randomly selected households were chosen from an exhaustive camp listing, used for voter registration and benefits, provided by the camp's Popular Committees. All household members 18 years of age or older were eligible, and were screened in the first phase by trained camp residents using structured questionnaires. For the second phase, a local psychologist reappraised a subset of participants.

\section{Phase 1}

Individuals could be assessed as screen-positive by either of two methods in phase 1: identified by a household informant (method A) or through direct interview (method B). In method A, a senior household representative was presented with a list of 
local terms and concepts (vignettes) for severe mental disorders. These vignettes were developed through community focus groups to ensure that distress in relation to severe disorders reflected the views of camp residents. Next, they were asked the five questions from the household informant portion of the World Health Organization - United Nations High Commissioner for Refugees (WHO-UNHCR) Assessment Schedule of Serious Symptoms in Humanitarian Settings (WASSS), an instrument designed and recommended for the detection of severe mental health and psychosocial problems in areas affected by disasters and armed conflict. ${ }^{13}$ Individuals were considered screen-positive if identified by a positive response to the vignettes or WASSS.

Those not screen-positive by method A were rescreened for common mental disorders by method B with the seven-question individual interview portion of the WASSS and the 20-item Self Reporting Questionnaire (SRQ); the latter is a mental health screening instrument developed by WHO to detect mental distress and common mental disorders across different cultures and has been internationally validated, including an Arabic version in Saudi Arabia and the United Arab Emirates. ${ }^{14,15}$ Participants were considered to have screened positive by this method if they responded positively ('all or most of the time') to any of the seven individual questions of WASSS or to a minimum of six questions in the SRQ. Additionally, participants were interviewed about sociodemographic and potential risk factors through translated and back-translated, piloted, structured questionnaires.

To maximise clinical assessments for those with psychiatric morbidity, all those screening positive by method $\mathrm{A}$, a randomly selected half of those screening positive by method B and a random $10 \%$ of those screening negative were asked to participate in phase 2 . These procedures also aimed to increase the probability of detecting more rare disorders and to minimise the burden of study-related home visits.

\section{Phase 2}

A clinical psychologist reassessed phase 2 participants for the following Axis I mental disorders using the Arabic version of the structured Mini International Neuropsychiatric Interview (MINI): ${ }^{16-18}$ major depressive episodes, dysthymia, suicidality, manic and hypomanic episodes, panic disorder, agoraphobia, social phobia, obsessive-compulsive disorder, PTSD, psychotic disorders, mood disorders with psychotic features and generalised anxiety disorder. Modules for alcohol and drug dependence, eating disorders and antisocial personality disorder were not included. Diagnoses and related periods (e.g. current, past, recurring episode) were assigned according to the instrument's instructions, which also take into account the rater's clinical judgement. The MINI has been validated against the longer Structured Clinical Interview for DSM Disorders, patient version (SCID-P) and the Composite International Diagnostic Interview for ICD-10 (CIDI). ${ }^{16,17}$ The Arabic version of the instrument showed good psychometric properties. ${ }^{18}$

Dysfunction was rated with the Global Assessment of Functioning (GAF) scale and the 12-item intervieweradministered version of the WHO Disability Assessment Schedule II (DAS-II). Both have been validated and are used extensively and internationally. ${ }^{19,20}$ The GAF is an Axis V (DSM-IV-TR) clinicianassessed rating of how well an individual meets problems in living, and ranges from 1 (complete impairment) to 100 (outstanding function); dysfunction was considered mild for scores 61-70, moderate for scores 51-60 and severe for scores lower than 51. ${ }^{19}$ The DAS-II corresponds to WHO criteria for the International Classification of Functioning and Disability, and measures limitations in cognition, mobility, self-care, getting along with others and participation in life activities on a self-reported five-point scale which is subsequently transformed into a global score ascending with degree of impairment. ${ }^{20}$ Given the high international variability of scores and in the absence of validated severity cut-offs for this community, the following scores were used: 5.6 (mild), 16.7 (moderate) and 41.7 (severe), respectively corresponding to the 50th, 75th and 90th percentiles of the weighted sample scores. This approach is consistent with recommendations to use 90th percentile scores and multiple thresholds for reporting disability. ${ }^{21,22}$ Reported dysfunction categories were based on meeting cut-off levels in either instrument. Additionally, phase 2 participants were asked about mental health service perceptions and utilisation and medication use through translated, back-translated, pilot-tested, structured questionnaires.

\section{Sample size}

Expecting at most 30\% mental disorder prevalence in our sample based on prior published estimates, ${ }^{8,23-25}$ a total of 896 phase 1 participants would be needed for a precision of $\pm 3 \%$ with $\alpha=0.05$. Assuming 3.6 eligible adults per household, ${ }^{26}$ and nonparticipation of $15 \%$ and $25 \%$ in the first and second phases respectively, we estimated needing 400 households. An additional 55 households were added to compensate for potentially untraceable households from the sampling list.

\section{Training and instrument pilot testing}

Interviewers were community members with prior experience in conducting surveys. Study staff received a week-long theoretical and practical training for obtaining informed consent, conducting the survey and for appropriate handling of human participants' research data. Four teams were each composed of an interviewer and supervisor, and were managed by an experienced study coordinator. The clinical psychologist who performed reappraisals in phase 2 was trained on study instruments and supervised by the study coordinator and referent psychiatrist. Study questions and procedures were pilot tested in the community prior to the study.

\section{Statistical analysis}

Population prevalence estimates, odds ratios (ORs) from logistic regression and corresponding robust confidence intervals were based on the phase 2 sample. These were probability weighted for differential selection procedures, participation rates, gender and age distribution differences against the source population as well as adjusted for clustering at household level. ${ }^{11,12}$ Sensitivity analyses considered $50 \%$ greater and lower probability of the outcome among non-participants per corresponding screening strata. Conditional probabilities and scores describing distribution of factors within categories or specific subgroups are presented as crude proportions, but as population estimates were weighted, crude and adjusted estimates may differ. Study data were double-entered using Epidata version 3.1 (Odense, Denmark) and analysed with Stata SE version 10.1 on Windows.

\section{Results}

\section{Phase 1}

Phase 1 participants were similar to the general camp population in terms of household composition, income and gender distribution, but were older owing to the study's age-related inclusion criteria (Table 1). Household representatives provided information for $748(83.5 \%)$ of the intended target of 896 persons (method A). From a refugee registry listing, 2251 households 455 were randomly selected, of which 40 were untraceable and 46 were 


\begin{tabular}{|c|c|c|c|}
\hline & All camp residents & Phase 1 & Phase 2 \\
\hline \multicolumn{4}{|l|}{ Households } \\
\hline Number of households & 3600 & 283 & 139 \\
\hline Size, $n$ : mean (range) & 5.3 & $4.3(1-8)$ & $4.7(1-8)$ \\
\hline Annual income, USD: mean (s.d.) & 3667-9642 & 4782 (2549) & $4558(2070)$ \\
\hline Age $\geqslant 18$ years, $n(\%)$ & $(60)^{a}$ & $748(61)^{b}$ & $381(62)^{\mathrm{C}}$ \\
\hline \multicolumn{4}{|l|}{ Individuals } \\
\hline Individuals, $n$ & $19000^{\mathrm{a}}$ & 748 & 194 \\
\hline Female, $n(\%)$ & 51.1 & $381(50.9)$ & $138(71.1)$ \\
\hline Age, years: mean (s.d.) & 29.8 & $39.0(15.8)$ & $41.5(15.1)$ \\
\hline $\begin{array}{l}\text { USD, US dollars. } \\
\text { a. Estimated from available information. }{ }^{10,11} \\
\text { b. Percentage of } 1222 \text { residents. } \\
\text { c. Percentage of } 616 \text { residents. }\end{array}$ & & & \\
\hline
\end{tabular}

empty; occupants were away from the camp in 21 households and were either repeatedly unavailable or refused participation in 65 households. Per protocol screening was completed for 561 individuals, $75 \%$ of 748 adults from the 283 participating households (method B). Screening procedures identified 51.2\% of adults potentially experiencing mental distress or disorders: $32.9 \%$ (246 of 748 ) by method A and $27.3 \%$ ( 86 of the 315 participants from the remaining 502) by method B (Fig. 1). The combined screening procedures detected current confirmed psychopathological disorder with $100 \%$ sensitivity and $62 \%$ specificity.

\section{Phase 2}

Phase 2 reappraisal was completed by 194 (59.5\%) of 326 selected individuals; these were more likely to be female $(P<0.001)$ and older $(P<0.01)$ than non-participants. Grouping all diagnoses, the prevalence of mental disorders was $29.0 \%$ (95\% CI $18.9-$ 39.2 ), and sensitivity analysis placed this estimate between $24.4 \%$ (95\% CI $19.3-29.6)$ and $39.4 \%$ (95\% CI 33.6-45.2). The prevalence of current mental disorders was $19.4 \%$ (95\% CI 12.6-26.2) and sensitivity analysis placed this estimate between $11.9 \%$ (95\% CI 8.5-15.3) and 25.7\% (95\% CI 20.7-30.7). Multiple diagnoses were noted in $42 \%(n=37)$ of the 88 persons with assessed mental disorder, largely consisting of combined mood and anxiety disorders $(57 \% ; n=21)$; almost all cases of psychosis (13 of 14) were also diagnosed with a mood or anxiety disorder. The distribution of mental disorders is given in Table 2. In ageand gender-adjusted logistic models mental disorders were associated with moderate to severe dysfunction ( $\mathrm{OR}=8.83,95 \%$ CI 4.47-17.42), whereas psychosis was associated with severe dysfunction (OR $=6.24,95 \%$ CI 1.97-19.75). Greatest impairment was noted in psychosis, mood disorders with psychotic features, social phobia, panic disorder, multiple diagnoses and mediumto high-risk suicidality, although absolute numbers were low (Table 3).

\section{Perception and use of services}

Of 88 persons in phase 2 with a psychiatric diagnosis, $5(6 \%)$ had received psychological or psychiatric care, corresponding to an adjusted treatment gap of $96 \%$ (95\% CI 92-100). These services were sought by $14 \%$ ( 2 of 14 ) of those diagnosed with psychosis and 3\% (2 of 60) of those with an Axis I disorder and moderate to severe dysfunction. Two-thirds of phase 2 respondents $(n=128)$ felt there was no appropriate access to mental health services, corresponding to a perceived treatment gap of $54 \%$ (95\% CI 41-68). Additionally, 40\% $(n=35)$ of those diagnosed had said they did not need mental health support, corresponding to a weighted $55 \%$ (95\% CI 38-72). Conversely, 15\% (16 of 106) of those not found to have an Axis I disorder felt they needed mental healthcare. Discordance between clinicians and participants in the
Table 2 Phase 2 Axis I diagnoses using the Mini International Neuropsychiatric Interview $(n=194)$

Crude, $n(\%) \quad$ Adjusted $\%(95 \% \mathrm{Cl})$

\begin{tabular}{|c|c|c|}
\hline \multicolumn{3}{|l|}{ Major depressive episode } \\
\hline Current & $31(16.0)$ & $8.3(4.4-12.2)$ \\
\hline Recurrent & $48(24.7)$ & $16.3(9.3-23.4)$ \\
\hline Lifetime & $61(31.4)$ & $19.3(11.7-26.8)$ \\
\hline Dysthymia, current & $8(4.1)$ & $1.4(0.4-2.5)$ \\
\hline \multicolumn{3}{|l|}{ Suicidality } \\
\hline Current & $24(12.4)$ & $8.1(3.8-12.4)$ \\
\hline Low risk & $18(9.3)$ & $6.2(2.8-9.6)$ \\
\hline Medium risk & $2(1.0)$ & $0.3(0.0-0.5)$ \\
\hline High risk & $4(2.1)$ & $1.7(0.0-4.1)$ \\
\hline \multicolumn{3}{|l|}{ Manic episode } \\
\hline Current & $3(1.5)$ & $1.1(0.0-2.4)$ \\
\hline Lifetime & $9(4.6)$ & $3.6(0.4-6.7)$ \\
\hline \multicolumn{3}{|l|}{ Hypomanic episode } \\
\hline Current & $2(1.0)$ & $0.4(0.0-1.0)$ \\
\hline Lifetime & $4(2.1)$ & $0.9(0.0-1.8)$ \\
\hline \multicolumn{3}{|l|}{ Panic disorder } \\
\hline Current & $8(4.1)$ & $5.9(0.0-12.7)$ \\
\hline Lifetime & $8(41.0)$ & $5.9(0.0-12.7)$ \\
\hline Agoraphobia, current & $4(2.1)$ & $1.0(0.0-2.2)$ \\
\hline Social phobia, current & $2(1.0)$ & $0.4(0.0-1.0)$ \\
\hline $\begin{array}{l}\text { Obsessive-compulsive disorder, } \\
\text { current }\end{array}$ & $7(3.6)$ & $2.2(0.0-4.4)$ \\
\hline PTSD, current & $9(4.5)$ & $2.2(0.5-3.9)$ \\
\hline \multicolumn{3}{|l|}{ Psychotic disorders } \\
\hline Current & $5(2.6)$ & $1.0(0.0-2.1)$ \\
\hline Lifetime & $14(7.2)$ & $3.3(1.0-5.5)$ \\
\hline $\begin{array}{l}\text { Mood with psychotic features, } \\
\text { current }\end{array}$ & $2(1.0)$ & $0.3(0.0-0.8)$ \\
\hline $\begin{array}{l}\text { Generalised anxiety disorder, } \\
\text { current }\end{array}$ & $15(7.7)$ & $1.0(0.0-2.0)$ \\
\hline \multicolumn{3}{|l|}{ All diagnoses } \\
\hline Current & 72 (37.1) & $19.4(12.6-26.2)$ \\
\hline Any & $88(45.4)$ & $29.0(18.9-39.2)$ \\
\hline Multiple & 37 (19.1) & $9.1(5.0-13.3)$ \\
\hline
\end{tabular}

phase 2 sample was $26 \%$, corresponding to a weighted population estimate of $21 \%$ (95\% CI 12-30). Of 194 phase 2 participants, 44 $(23 \%)$ reported present $(n=21)$ or past $(n=30)$ psychotropic use, corresponding to a weighted frequency of $11 \%$ (95\% CI 6-16). These medications comprised antidepressants $(n=10)$, anxiolytics $(n=5)$, opioid-based analgesics $(n=3)$, antipsychotics $(n=1)$, mood stabilisers $(n=1)$ and sleeping aids $(n=1)$. The majority 


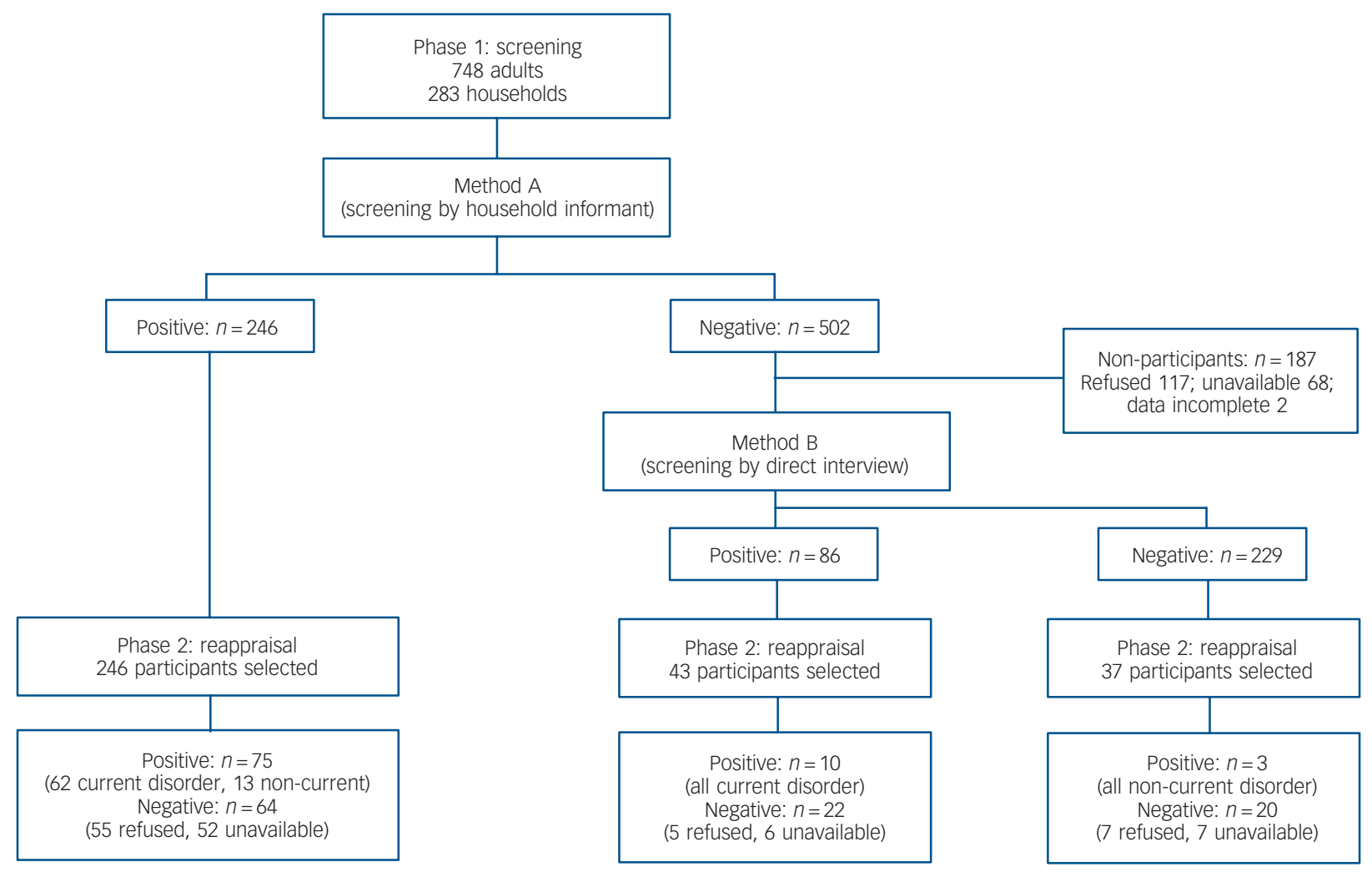

\section{Fig. 1 Study profile.}

\section{Table 3 Distribution of dysfunction scores by diagnosis}

\begin{tabular}{|c|c|c|c|c|c|}
\hline & \multirow[b]{2}{*}{$n$} & \multicolumn{2}{|c|}{ Median dysfunction } & \multicolumn{2}{|c|}{ GAF or DAS-II, \% ${ }^{\mathrm{a}}$} \\
\hline & & GAF & DAS-II & $\geqslant$ Moderate & Severe \\
\hline \multicolumn{6}{|l|}{ Psychotic disorders } \\
\hline Psychotic disorders & 14 & Moderate & Moderate & 93 & 43 \\
\hline 0-1 comorbidity & 10 & Moderate & Moderate & 90 & 30 \\
\hline 2-3 comorbidities & 4 & Moderate & Severe & 100 & 75 \\
\hline \multicolumn{6}{|l|}{ Mood disorders } \\
\hline \multicolumn{6}{|l|}{ Mood disorder without psychosis } \\
\hline Major depressive disorder & 40 & Moderate & Moderate & 68 & 30 \\
\hline Dysthymia & 8 & Mild & Moderate & 88 & 38 \\
\hline Bipolar disorder ${ }^{b}$ & 10 & Mild & Mild & 50 & 40 \\
\hline Mood disorder with psychotic features & 2 & Moderate & Moderate & 100 & 50 \\
\hline Any mood disorder & 58 & Moderate & Moderate & 67 & 33 \\
\hline Mood disorder without comorbid anxiety disorder & 37 & Mild & Moderate & 60 & 27 \\
\hline \multicolumn{6}{|l|}{ Anxiety disorders } \\
\hline Panic disorder & 7 & Moderate & Severe & 71 & 57 \\
\hline Agoraphobia & 3 & Mild & Moderate & 67 & 33 \\
\hline Social phobia & 2 & Moderate & Severe & 100 & 100 \\
\hline Obsessive-compulsive disorder & 5 & Moderate & Moderate & 80 & - \\
\hline PTSD & 8 & Moderate & Moderate & 88 & 38 \\
\hline Generalised anxiety disorder & 13 & Mild & Moderate & 62 & 15 \\
\hline Any anxiety disorder & 32 & Moderate & Moderate & 72 & 28 \\
\hline Anxiety disorder without comorbid mood disorder & 11 & Mild & Mild & 55 & \\
\hline \multicolumn{6}{|l|}{ Suicidality } \\
\hline Low risk & 18 & Moderate & Moderate & 78 & 33 \\
\hline Medium or high risk & 6 & Moderate & Moderate & 67 & 50 \\
\hline Any suicide risk & 24 & Moderate & Moderate & 75 & 38 \\
\hline \multicolumn{6}{|l|}{ Mental health disorders } \\
\hline None & 106 & $<$ Mild & $<$ Mild & 25 & 5 \\
\hline Any mental disorder & 88 & Moderate & Moderate & 68 & 28 \\
\hline Any current mental disorder & 72 & Moderate & Moderate & 73 & 32 \\
\hline 0-1 comorbidity & 80 & Moderate & Moderate & 65 & 25 \\
\hline 2-3 comorbidities & 8 & Moderate & Severe & 100 & 63 \\
\hline
\end{tabular}


( $n=18)$ of the 21 participants currently using medication were also diagnosed with a mental disorder in phase 2 , and 17 reported having a medical prescription for their use.

\section{Discussion}

This study contributes clinically confirmed prevalence estimates for a range of mental disorders, associated disability and treatment gap for adults in the long-term refugee setting of the Burj el-Barajneh camp in Lebanon. To the best of our knowledge this is the first population-based study to assess severe mental disorders in a refugee camp and the second to do so in a humanitarian setting. ${ }^{27}$ Methodological and contextual differences limit comparability of mental disorder prevalence rates across studies and populations. ${ }^{28,29}$ In our study the overall prevalence of current mental disorders was $19.4 \%$ (95\% CI 12.6-26.2); the range of global 12-month estimates is $6-27 \%$ (interquartile range $9.8-19.1$ ). ${ }^{30}$

The frequency of depression and PTSD is typically elevated in conflict-affected groups. Here, the lifetime occurrence of major depressive episodes (19.3\%, 95\% CI 11.7-26.8) was closest to that noted in post-conflict settings with comparable adversity characteristics $(16 \%),{ }^{29}$ whereas that of current PTSD $(2.2 \%$, 95\% CI 0.5-3.9) was most similar to 12-month background levels reported in Lebanon and the EU $(2-5 \%) \cdot{ }^{10,29,31,32}$ Estimates from Sri Lanka and the Occupied Palestinian Territories suggest that the prevalence of both PTSD and depression may remain elevated in ongoing conflicts. ${ }^{23,33}$ In contrast, the pattern observed in our study may reflect a natural course in residents of long-term refugee camps, settings characterised by lower exposure to adversity but high in daily life stressors related to poverty and marginalisation.

Although the lifetime prevalence of psychosis (3.3\%, 95\% CI 1.0-5.5) fell within the range of estimates from studies with similar methodology $(0.3-3.5 \%)$, the definitions used varied considerably. ${ }^{34-37}$ The prevalence of active psychosis (1\%, 95\% CI 0-2) was consistent with estimates in the two-phase study conducted in post-conflict East Timor (Timor Leste), which estimated a nonaffective psychosis point prevalence of $1.2 \%{ }^{32}$ Only a small minority of those with a history of psychosis, despite its association with high levels of dysfunction, had accessed mental health services.

Despite well-promoted, freely available services, the mental health treatment gap inside the camp was $96 \%$ (95\% CI 92-100) compared with the Lebanese national figure of $51 \% .^{25}$ This, together with a meaningful discordance between clinician diagnosis and self-perceived need for care, and the known stigmatisation of mental disorders in the camp, pose a challenge to improving service utilisation.

\section{Study limitations}

This study has several limitations. Non-participation was greater than expected, particularly among young men. Imbalances were addressed through post-stratification weights and a sensitivity analysis; the low sample size is reflected by wide confidence intervals around our estimates. Absence and household untraceability may have resulted in selection bias, although neither is likely to be related to the outcome of interest. The household listing was provided by the camp's Popular Committees and is believed to be fairly complete as it is used for voting and refugee benefits. Results are thus likely to be generalisable to the study population: adults 18 years of age and older living in the camp.

The MINI does not cover all mental disorders. Additionally, some modules were excluded in this study. Eating disorders were felt to be rare in this context, personality disorders were deemed difficult to measure in a single session and substance use disorders were not therapeutically supported by the organisation's free mental health services. It is probable, however, that where present these conditions would coexist with assessed disorders. Nonetheless, our overall mental disorder prevalence estimates may be low. Furthermore, reappraisals were performed by a single psychologist, thus interrater reliability could not be assessed, and although questions on healthcare use were pilot-tested, they were not validated. It would be worthwhile for future investigations to include a broader scope of disorders, especially substance misuse, to understand their specific prevalence and interaction with others, as well as to employ more than one assessor and contrast their findings, and include context-specific, validated questions and instruments where possible.

Close to half of those with diagnosed psychopathological disorder expressed a feeling that they had no need of mental healthcare. This may question the cultural relevance of our assessments and cultural conceptions of mental health. The MINI, used here for diagnosis, has been internationally validated against longer DSM and ICD structured interview instruments and the Arabic version has shown good psychometric properties. ${ }^{18,38}$ Furthermore, the clinical interviews were conducted by a local research-trained, licensed psychologist familiar with the culture and context as well as supported by a senior psychiatrist. Lastly, dysfunction was significantly predicted by mental disorders and showed a severity gradient, suggesting that the diagnoses are relevant in this context. Nevertheless, this meaningful mismatch between clinician and patient understanding of mental health is probably tied to cultural expression, beliefs and stigma, and has major implications for improving health-seeking behaviour and reducing the treatment gap.

\section{Meeting healthcare needs}

Although healthcare is free and well-promoted within Burj el-Barajneh camp, the level of unmet need remains a challenge. Reducing the treatment gap necessitates a comprehensive approach which includes sensitisation, training, supervision and appropriate diagnosis and treatment. The approach taken by MSF mental health programmes in Burj el-Barajneh and subsequently in Ain al-Hilweh has been multicomponent. First, positive mental health is promoted through community awareness programmes and sensitisation of primary healthcare staff. As the programme is integrated into existing primary health structures, it takes advantage of additional opportunities to provide information and awareness in a neutral setting. The cornerstone of the approach is the provision of continual training and supervision, which encourages capacity building and timely and appropriate diagnosis and treatment. By encouraging non-governmental organisations (NGOs) and primary healthcare staff to work together as a team, this multidisciplinary approach, which includes psychiatrists, psychologists, psychiatric nurses and social workers, facilitates intervention. The NGO staff provide training and side-by-side mentoring on site, which helps to ensure that disorders are detected and referred when needed, and that less complex presentations are addressed per Mental Health Gap Action Programme guidelines. ${ }^{39}$ Training and supervision of NGO staff are conducted by a senior psychiatrist through individual and team meetings and case reviews.

\section{Implications of the study}

Our study's two-phase strategy allowed estimation of the prevalence of a range of mental disorders devoid of the usual conflation by distress shown in single-phase studies with layadministered questionnaires. We found that more than a fifth of adults in this protracted refugee setting had at least one mental disorder, and that these conditions were often comorbid, disabling and largely untreated. Our findings add rare evidence to the 
current consensus-based policy to prioritise availability of mental health treatment in refugee settings, especially for the most severe and disabling conditions. ${ }^{4,40}$ Our study also highlights the importance of understanding mental health-related cultural beliefs and barriers to accessing available support towards improving the provision of appropriate, acceptable and effective care.

\footnotetext{
Augusto E. Llosa, PhD, Epicentre, Paris, France; Zeina Ghantous, MPH, Médecins Sans Frontières, Geneva, Switzerland; Renato Souza, MD, Médecins Sans Frontières, Geneva, Switzerland, and Department and Institute of Psychiatry. Faculty of Medicine, University of São Paulo, Brazil; Fabio Forgione, MSC, Pierre Bastin, MD, Alison University of São Paulo, Brazil; Fabio Forgione, MSC, Pierre Bastin, MD, Alison,
Jones, MSC, Annick Antierens, MD, Andrei Slavuckij, MD, Médecins Sans Jones, MSC, Annick Antierens, MD, Andrei Slavuckij, MD, Médecins Sans
Frontières, Geneva, Switzerland; Rebecca. F. Grais, PhD, Epicentre, Paris, France

Correspondence: Dr Augusto Llosa, Department of Epidemiology and Population Health, Epicentre, 8 Rue Saint Sabin, Paris 75011, France. Email: Augusto.LLOSA@epicentre.msf.org

First received 5 Sep 2012, final revision 17 Apr 2013, accepted 8 May 2013
}

\section{Funding}

The study was funded by Médecins Sans Frontières.

\section{Acknowledgements}

We thank Dr Samar Zebian, Dr Rima Afifi, Dr Mona Osman and Dr Vikram Patel for advice the conduct of the study, and the residents of Burj el-Barajneh refugee camp for their agreement and support in conducting this research.

\section{References}

1 United Nations High Commissioner for Refugees. A Year of Crises: UNHCR Global Trends 2011. UNHCR, 2012.

2 Reed RV, Fazel M, Jones L, Panter-Brick C, Stein A. Mental health of displaced and refugee children resettled in low-income and middle-income countries: risk and protective factors. Lancet 2012; 379: 250-65.

3 Fazel M, Reed RV, Panter-Brick C, Stein A. Mental health of displaced and refugee children resettled in high-income countries: risk and protective factors 2. Lancet 2012; 379: 266-82.

4 Jones L, Asare JB, El Masri M, Mohanraj A, Sherief $H$, van Ommeren M. Severe mental disorders in complex emergencies. Lancet 2009; 374: 654-61.

5 Miller KE, Rasmussen A. War exposure, daily stressors, and mental health in conflict and post-conflict settings: bridging the divide between traumafocused and psychosocial frameworks. Soc Sci Med 2010; 70: 7-16.

6 Saxena S, Thornicroft G, Knapp M, Whiteford H. Resources for mental health: scarcity, inequity, and inefficiency. Lancet 2007; 370: 878-89.

7 Chaaban J, Ghattas H, Habib R, Hanafi S, Sahyoun N, Salti N, et al. Socio-Economic Survey of Palestinian Refugees in Lebanon. American University of Beirut, 2010.

8 Tiltnes A. Falling Behind. A Brief on the Living Conditions of Palestinian Refugees in Lebanon. Fafo Institute for Applied Social Science, 2005

9 Chahine L, Chemali Z. Mental health care in Lebanon: policy, plans and programmes. East Mediterr Health J 2009; 15: 1596-612.

10 Tol WA, Barbui C, Galappatti A, Silove D, Betancourt TS, Souza R, et al. Mental health and psychosocial support in humanitarian settings: linking practice and research. Lancet 2011; 378: 1581-91.

11 Dunn G, Pickles A, Tansella M, Vázquez-Barquero JL. Two-phase epidemiological surveys in psychiatric research. Br J Psychiatry 1999; 174: 95-100.

12 Pickles A, Dunn G, Vazquez-Barquero JL. Screening for stratification in two-phase ('two-stage') epidemiological surveys. Stat Methods Med Res 1995; 4: 73-89.

13 World Health Organization, United Nations High Commissioner for Refugees. WHO-UNHCR Assessment Schedule of Serious Symptoms in Humanitarian Settings (WASSS) (Field-Test Version). In Assessing Mental Health and Psychosocial Needs and Resources: Toolkit for Major Humanitarian Crises: 34-40. WHO/UNHCR, 2012.

14 Al-Subaie AS, Mohammed K, Al-Malik T. The Arabic self-reporting questionnaire (SRQ) as a psychiatric screening instrument in medical patients. Ann Saudi Med 1998; 18: 308-10.

15 El-Rufaie OEF, Absood GH. Validity study of the Self-Reporting Questionnaire (SRQ-20) in primary health care in the United Emirates. Int J Methods Psychiatr Res 1994; 4: 45-53.

16 Sheehan DV, Lecrubier $\mathrm{Y}$, Sheehan $\mathrm{KH}$, Amorim P, Janavs J, Weiller E, et al. The Mini-International Neuropsychiatric Interview (M.I.N.I.): the development and validation of a structured diagnostic psychiatric interview for DSM-IV and ICD-10. J Clin Psychiatry 1998; 59 (suppl 20): 22-33.
17 Sheehan D, Lecrubier Y, Harnett-Sheehan K, Janavs J, Weiller E, Keskiner A, et al. Reliability and validity of the MINI International Neuropsychiatric Interview (M.I.N.I.): according to the SCID-P. Eur Psychiatry 2011; 12: 232-41.

18 Kadri N, Agoub M, El Gnaoui S, Alami KM, Hergueta T, Moussaoui D. Moroccan colloquial Arabic version of the Mini International Neuropsychiatric Interview (MINI): qualitative and quantitative validation. Eur Psychiatry 2005; 20: 193-5.

19 Hilsenroth MJ, Ackerman SJ, Blagys MD, Baumann BD, Baity MR, Smith SR, et al. Reliability and validity of DSM-IV axis V. Am J Psychiatry 2000; 157: 1858-63.

20 Luciano JV, Ayuso-Mateos JL, Aguado J, Fernandez A, Serrano-Blanco A, Roca M, et al. The 12-item World Health Organization Disability Assessment Schedule II (WHO-DAS II): a nonparametric item response analysis. BMC Med Res Methodol 2010; 10: 45.

21 Mont D. Measuring Disability Prevalence. World Bank, 2007

22 Von Korff M, Crane PK, Alonso J, Vilagut G, Angermeyer MC, Bruffaerts R, et al. Modified WHODAS-II provides valid measure of global disability but filter items increased skewness. J Clin Epidemiol 2008; 61: 1132-43.

23 Canetti D, Galea S, Hall BJ, Johnson RJ, Palmieri PA, Hobfoll SE. Exposure to prolonged socio-political conflict and the risk of PTSD and depression among Palestinians. Psychiatry 2010; 73: 219-31.

24 Karam EG, Mneimneh ZN, Karam AN, Fayyad JA, Nasser SC, Chatterji S, et al. Prevalence and treatment of mental disorders in Lebanon: a national epidemiological survey. Lancet 2006; 367: 1000-6.

25 Karam EG, Mneimneh ZN, Dimassi H, Fayyad JA, Karam AN, Nasser SC, et al. Lifetime prevalence of mental disorders in Lebanon: first onset, treatment, and exposure to war. PLOS Med 2008; 5: e61.

26 United Medical Group. Mental Health Among Women and Adolescents in the Palestinian Refugee Camps in Lebanon. UMG, 2006.

27 Silove D, Bateman CR, Brooks RT, Fonseca CA, Steel Z, Rodger J, et al. Estimating clinically relevant mental disorders in a rural and an urban setting in postconflict Timor Leste. Arch Gen Psychiatry 2008; 65: 1205-12.

28 Fazel M, Wheeler J, Danesh J. Prevalence of serious mental disorder in 7000 refugees resettled in western countries: a systematic review. Lancet 2005; 365: 1309-14

29 Steel Z, Chey T, Silove D, Marnane C, Bryant RA, van Ommeren M. Association of torture and other potentially traumatic events with mental health outcomes among populations exposed to mass conflict and displacement: a systematic review and meta-analysis. JAMA 2009; 302: 537-49.

30 Kessler RC, Aguilar-Gaxiola S, Alonso J, Chatterji S, Lee S, Ormel J, et al. The global burden of mental disorders: an update from the WHO World Mental Health (WMH) surveys. Epidemiol Psichiatr Soc 2009; 18: 23-33.

31 De Jong JT, Komproe IH, van Ommeren M. Common mental disorders in postconflict settings. Lancet 2003; 361: 2128-30

32 Darves-Bornoz JM, Alonso J, de Girolamo G, de Graaf R, Haro JM, Kovess-Masfety $\mathrm{V}$, et al. Main traumatic events in Europe: PTSD in the European study of the epidemiology of mental disorders survey. J Trauma Stress 2008; 21: 455-62.

33 Husain F, Anderson M, Lopes Cardozo B, Becknell K, Blanton C, Araki D, et al. Prevalence of war-related mental health conditions and association with displacement status in postwar Jaffna District, Sri Lanka. JAMA 2011; 306 522-31.

34 Gureje O, Olowosegun O, Adebayo K, Stein DJ. The prevalence and profile of non-affective psychosis in the Nigerian Survey of Mental Health and Wellbeing. World Psychiatry 2010; 9: 50-5.

35 Kessler RC, Birnbaum H, Demler O, Falloon IR, Gagnon E, Guyer M, et al. The prevalence and correlates of nonaffective psychosis in the National Comorbidity Survey Replication (NCS-R). Biol Psychiatry 2005; 58: 668-76.

36 Ochoa S, Haro JM, Torres JV, Pinto-Meza A, Palacin C, Bernal M, et al. What is the relative importance of self reported psychotic symptoms in epidemiological studies? Results from the ESEMeD-Catalonia Study. Schizophr Res 2008; 102: 261-9.

37 Perala J, Suvisaari J, Saarni SI, Kuoppasalmi K, Isometsa E, Pirkola S, et al. Lifetime prevalence of psychotic and bipolar I disorders in a general population. Arch Gen Psychiatry 2007; 64: 19-28.

38 Lecrubier Y, Sheehan DV, Weiller E, Amorim P, Bonora I, Harnett K, et al. The MINI International Neuropsychiatric Interview (M.I.N.I.): a short diagnostic structured interview: reliability and validity according to the CIDI. Eur Psychiatry 2011; 12: 224-31.

39 World Health Organization. mhGAP: Mental Health Gap Action Programme: Scaling Up Care for Mental, Neurological and Substance Use Disorders. WHO, 2008.

40 Inter-Agency Standing Committee. IASC Guidelines on Mental Health and Psychosocial Support in Emergency Settings. IASC, 2007 (http:// www.who.int/mental_health/emergencies/guidelines_iasc_mental_health psychosocial_june_2007.pdf). 